\section{Streptococcus pneumoniae intracranial abscess and post-infectious vasculitis}

\section{Alexandra Lucas, ${ }^{1}$ Ko Ko Maung, ${ }^{2}$ Ryan Ratts ${ }^{3}$}

${ }^{1}$ Geisel School of Medicine at Dartmouth, Hanover, NH; 'Department of Medicine, Dartmouth-Hitchcock Medical Center, Lebanon, NH; ${ }^{3}$ Department of Hospital Medicine, Dartmouth-Hitchcock Medical Center, Lebanon, NH, USA

\begin{abstract}
Intracranial abscesses are rare complications of Streptococcus pneumoniae infections, and to our knowledge, there have been no case reports of post-infectious vasculitis developing in such patients. Here we describe the case of a 48-year-old post-splenectomy male who developed post-infectious vasculitis following $S$. pneumoniae otitis media complicated by mastoiditis, osteomyelitis, meningitis, and intracranial abscess. Clinicians ought to be aware of the possible adverse outcomes of invasive $S$. pneumoniae and the limitations of current treatment options.
\end{abstract}

\section{Introduction}

Streptococcus pneumoniae intracranial abscess is a rare complication of pneumococcal infections, with the majority of cases being reported in the pre-antibiotic era. Today, the survival rate and long-term outcome of these patients remains quite poor, with up to $35 \%$ mortality and $40 \%$ experiencing prolonged neurological deficits. ${ }^{1,2}$ Most infections occur as a result of contiguous infection, with paranasal sinus and the middle ear being the most common sources. ${ }^{3}$ Post-infectious vasculitis is an uncommon complication of $S$. pneumoniae, with arterial cerebrovascular complications occurring in up to $21.8 \%$ of $S$. pneumoniae meningitis. ${ }^{4}$ To our knowledge, no case report has identified post-infectious vasculitis occurring after $S$. pneumoniae abscess in an adult. Here, we report a case of a $48 \mathrm{yo}$ male who developed post-infectious vasculitis following $S$. pneumoniae otitis media, mastoiditis, osteomyelitis, meningitis and abscess.

\section{Case Report}

Our patient is a 48 year-old male patient with childhood splenectomy and no pneumococcal vaccine who initially presented to an outside emergency department (ED) with ear pain and headache. He was given amoxicillin and hydrocodone bitartrate plus acetaminophen and was discharged home. That night he developed altered mental status, becoming uncommunicative and unable to follow commands. He re-presented to the same ED and found to be hypertensive and febrile, with a Glascow coma scale (GCS) score of 13 without focal motor or sensory deficits. A complete blood count revealed leukocytosis with a white blood cell (WBC) count of $51 \times 10^{3} / \mathrm{mcL}$ (reference value $4.0-10.0 \times 10^{3} / \mathrm{mcL}$ ). An MRI demonstrated a left $4 \times 4 \times 3 \mathrm{~cm}$ heterogenous multiloculated mass extending intracranially from the mastoid air cells, consistent with mastoid abscess and osteomyelitis (Figure 1A). An epidural abscess, sigmoid sinus and jugular thrombosis were also seen (Figure 1B). Lumbar puncture showed 2600 white blood cells per mcl (reference value $0-5 / \mathrm{mcL}$ ), 250 red blood cells per mcL (reference value 0 $5 / \mathrm{mcL}$ ), total protein content of $924 \mathrm{mg} / \mathrm{dL}$ (reference value $15-45 \mathrm{mg} / \mathrm{dL}$ ), glucose $<1 \mathrm{mg} / \mathrm{dL}$. Gram staining revealed neutrophils and grampositive diplococci. He was transferred to our hospital, where he was taken to the operating room for mastoidectomy, craniotomy, and CSF shunt placement with evacuation of intradiploic skull mass and epidural abscess. Pathology of skull mass demonstrated a benign epithelial inclusion cyst with lymphocytic infiltrate. Initial antibiotic regimen included IV vancomycin, ceftriaxone, and steroid regimen of IV dexamethasone. Mastoid fluid culture revealed $S$. pneumoniae resistant to penicillin and ceftriaxone (unusual in our institution) and ceftriaxone was changed to rifampin. His status improved over the next 8 days, and he was discharged on hospital day (HD) 8 with an ongoing headache well controlled with oxycodone, a Montreal Cognitive Assessment (MOCA) of 19/30, and a decreasing leukocytosis (white blood cell count $20.2 \times 10^{3} / \mathrm{mcL}$ ). At discharge, he remained on IV vancomycin and P0 rifampin for a planned six-week course, and a two week P0 dexamethasone taper starting from 10mg PO bid.

Two days after discharge, he re-presented to the outside ED with worsening temporal headache. His neurological exam was normal, but he was febrile to $38.2 \mathrm{C}$. A complete blood cell count revealed $22.6 \times 10^{3} / \mathrm{mcL}$ white blood cells, platelets $900 \times 10^{3} / \mathrm{mcL}$ (reference 145 $370 \times 10^{3} / \mathrm{mcL}$ ), and a new anemia, with hemoglobin of $11.4 \mathrm{gm} / \mathrm{dL}$ (reference range 13.7$17.5 \mathrm{gm} / \mathrm{dL}$ ). A CT head revealed persistent opacification of residual left mastoid. He was begun on levofloxacin in addition to his vancomycin and rifampin, and transferred to our hospital. An MRI obtained at our institution demonstrated increased meningeal enhance-
Correspondence: Alexandra Lucas, 30 Wolf Road Apt 213, Lebanon, NH 03766, USA.

Tel.: + 1.978.771.9665.

E-mail: alexandra.t.lucas.med@Dartmouth.edu

Key words: streptococcus pneumoniae, intracranial abscess, post-infectious vasculitis.

Contributions: the authors contributed equally.

Conflict of interest: the authors declare no potential conflict of interest.

Received for publication: 22 August 2016.

Revision received: 3 November 2016.

Accepted for publication: 2 December 2016.

This work is licensed under a Creative Commons Attribution-NonCommercial 4.0 International License (CC BY-NC 4.0).

(C) Copyright A. Lucas et al., 2016

Licensee PAGEPress, Italy

Infectious Disease Reports 2016; 8:6844

doi:10.4081/idr.2016.6844

ment and new left cerebellar enhancement that was consistent with either post-surgical change or cerebellitis. His LP was consistent with persistent signs of infection versus inflammation (WBC 846 cells per mcL, protein $168 \mathrm{mg} / \mathrm{dL}$, and glucose $4 \mathrm{mg} / \mathrm{dL}$ ). His IV dexamethasone was empirically increased to 10 mg IV. On HD2, he developed sudden-onset of slurred speech and right facial droop; a CT did not demonstrate any new infarcts. Over the next 3 days, he remained febrile, dysarthric, confused, and agitated. His dexamethasone was decreased to $6 \mathrm{mg}$ and then $4 \mathrm{mg}$ bid to allow for better penetration of antibiotics. Given his ongoing signs of infection, ceftaroline was added to his regimen and levofloxacin replaced by moxifloxacin. Multiple blood and CSF cultures demonstrated no growth. On HD10, a stroke alert was activated due to unresponsiveness to voice and sternal rub. A CT head demonstrated a subacute lacunar infarct of the left basal ganglia and corona radiate, and levetiracetam was begun for seizure prophylaxis. An MRI the following day showed several punctate areas of restricted diffusion, most likely small areas of infarct consistent with post-infectious vasculitis (Figure 2). His dexamethasone was increased to $8 \mathrm{mg}$ IV q8h, and his obtundation improved the next day. No intravenous immunoglobulin (IVIG) was given. Over the course of the next 47 days, he had fluctuating mental status that gradually improved. Consulting services during admission included neurosurgery, ENT, infectious disease, and neurology. His course was complicated by thrush, red man syndrome, hyponatremia secondary to SIADH, and urinary reten- 

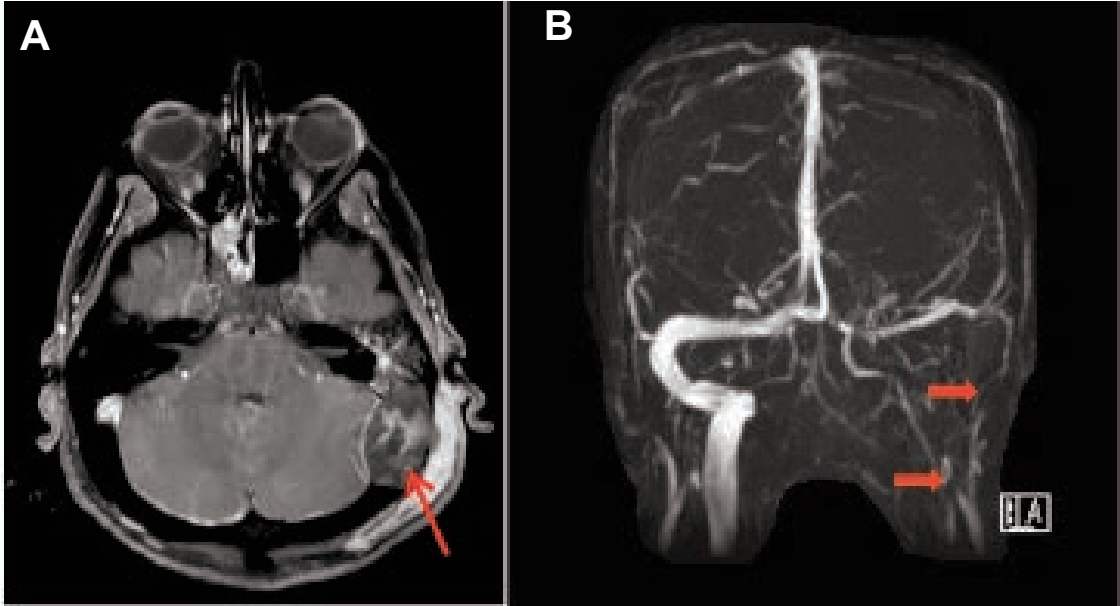

Figure 1. Patient MRA obtained on HD1 of first admission. A) T1-weighted precontrast imaging demonstrating a $3 \times 4 \mathrm{~cm}$ area of high signal intensity extending posteriorly from the left mastoid, consistent with mastoid abscess versus osteomyelitis. B) MRV demonstrating sigmoid sinus and jugular thrombosis.

tion. He was maintained on a prolonged steroid taper and completed an additional 4 week course of vancomycin, rifampin, ceftaroline, and moxifloxacin. On HD46, he underwent an interventional radiology-guided mastoid fluid drainage, which showed only few WBCs without organisms or growth. On HD57, he was discharged home following an MRI that showed evolution of his old infarcts and no new disease. Post-discharge, his cognition, memory and motor function continued to gradually improved. Unfortunately, he died a few months later as a passenger in a single motor vehicle accident before full neurological recovery was achieved.

\section{Discussion}

This complex case highlights the risk of vascular involvement in invasive pneumococcal infections. Patients with $S$. pneumoniae infections are at particular risk of such complications given the pro-inflammatory cascade triggered by the bacteria, regardless of initial antibiotic therapy. ${ }^{5}$ Markers of both neuronal, glial, and myelin destruction have been identified in $S$. pneumoniae meningitis patients, likely due to streptococcal cell wall induced cytokine toxicity. ${ }^{6}$

Our patient was at higher risk of invasive $S$. pneumoniae infection given his history of splenectomy without pneumococcal vaccination and of benign epithelial inclusion cyst. Underlying chronic predispositions for infection are found in over $40 \%$ of patients, with chronic alcoholism, asplenia, diabetes mellitus, and malignancy being among the most common. ${ }^{4,7}$ The majority of patients present with an associated infection, mainly ear or sinus infections or pneumonias. ${ }^{4}$ Nonetheless, pneumococcal abscesses remain very rare, with the majority of reported cases occurring in the pre-antibiotic era.

Given the rarity of post-infectious vasculitis, there is no consensus on treatment for $S$. pneumoniae cerebral vasculitis. Corticosteroids are effective in preventing complications of meningitis, e.g. sensorineural hearing loss, if given very early in the course of treatment (prior to or at the time of antibiotics). Although treatment is typically limited to less than four days, some reports have suggested a rebound inflammatory effect whereby steroid withdrawal can result in increased vascular inflammation. ${ }^{5}$ Our patient's course adds to this growing body of literature, given his progression while on a prolonged taper of steroids. The role of IVIG is not well-established in this population, although it is used in isolated cases of non-infectious cerebral vasculitis. However, IVIG and glucocorticoids have been reported to be efficacious in patients with post-infectious vasculitis. ${ }^{6}$ Although our patient did not receive IVIG, this is a possible treatment option that ought to be considered.

\section{Conclusions}

This report highlights the post-infectious vascular complications of invasive pneumococcal disease in an at-risk patients. Clinicians should be aware of the severity of illness and the possible adverse outcomes of $S$. pneumoni$a e$. More research needs to be done in order to establish the various roles of antibiotics, glucocorticoids, and IVIG treatments so that we can effectively manage these patients in the future.

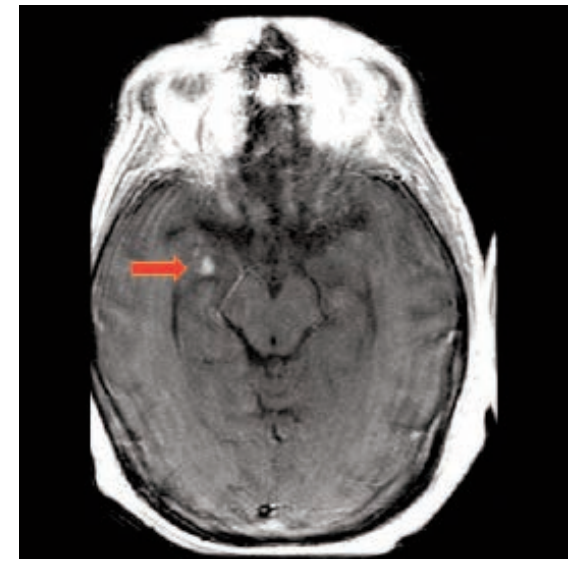

Figure 2. T2-weighted MRI obtained on HD11 of second admission, demonstrating punctate areas of restricted diffusion, most likely small areas of infarct consistent with post-infectious vasculitis.

\section{References}

1. Grigoriadis E, Gold WL. Pyogenic brain abscess caused by streptococcus pneumonia: case report. Clin Infect Dis 1997;25:1108-12.

2. Tseng JH, Tseng MY. Brain abscess in 142 patients: factors influencing outcome and mortality. Surg Neurol 2006;65:557-562.

3. Carpenter J, Stapleton S, Holliman R. Retrospective analysis of 49 cases of brain abscess and review of the literature. Eur $\mathrm{J}$ Clin Microbiol Infect Dis 2007;26:1-11.

4. Kastenbauer S, Pfister HW. Pneumococcal meningitis in adults: spectrum of complications and prognostic factors in a series of 87 cases. Brain 2003;126:1015-25.

5. Ribeiro S, Domingues V, Faria RM, Mendonça T. Invasive pneumococcal disease complicated by cerebral vasculitis, transient diabetes inspidus and spondylodiscitis. BMJ Case Rep 2013:bcr2013010336.

6. Jorens PG, Parizel PM, Demey HE, et al. Meningoencephalitis cased by streptococcus pneumoniae: a diagnostic and therapeutic challenge. Neuroradiology 2005;47:758-64.

7. Rueda AM, Serpa JA, Matloobi M, et al. The spectrum of invasive pneumonoccal disease at an adult tertiary care hospital in the early $21^{\text {st }}$ century. Medicine 2010;89:331.

8. Lu CH, Chang WN, Lui CC. Strategies for the management of bacterial brain abscess. $\mathrm{J}$ Clin Neurosci 2006;13:979-85.

9. Xiao F, Tseng MY, Teng LJ, et al. Brain abscess: clinical experience and analysis of prognostic factors. Surg Neurol 2005;63:44250.

10. Hakan T, Ceran N, Erdem I, et al. Bacterial brain abscesses: an evaluation of 96 cases. J Infect 2006;52:359-66. 